\title{
AURICULOTERAPIA NO TRATAMENTO DO ZUMBIDO: ESTUDO DE CASOS
}

\author{
AURICULOTHERAPY IN THE TREATMENT OF TINNITUS: CASE \\ STUDY
}

\author{
Ana Lúcia Basílio Carneiro ${ }^{1}$ \\ Mariana Nunes Azevedo de Melo ${ }^{2}$ \\ Lindair Alves da Silva ${ }^{3}$
}

RESUMO: OBJETIVO: Verificar a ação da auriculoterapia em pacientes com queixa de zumbido. MÉTODO: Estudo de intervenção, com avaliação pré e pós sessões de auriculoterapia, apresentado como estudo de caso em três pacientes com queixa de zumbido. Foram realizadas avaliações com o Tinnitus Handicap Inventory (THI), a Escala Analógica Visual (EVA), o Inventário de Ansiedade Traço-Estado (IDATE) e inventário de ansiedade Beck (Beck Anxiety Inventory - BAI) antes e depois de oito sessões de auriculoterapia. A avaliação auricular foi realizada por inspeção visual, eletrodiagnóstico e palpação. Após diagnóstico energético e auricular foi determinado a combinação de pontos para cada paciente. Em todos os protocolos foram incluídos os pontos Shen men, rim, ouvido interno, ouvido externo, tronco cerebral, tálamo e temporal. Para compor o protocolo individual, outros pontos foram selecionados considerando o zumbido um sintoma multifatorial e o cuidado integral do paciente. RESULTADOS: Os dados coletados nos inventários e os relatos dos pacientes demonstraram que a auriculoterapia reduziu os sintomas do zumbido e da ansiedade e melhorou a qualidade de vida dos pacientes. CONCLUSÃO: A auriculoterapia é uma tecnologia de cuidado integral que altera a percepção do zumbido e melhora a qualidade de vida dos pacientes, mostrando-se uma intervenção promissora e eficiente nos casos avaliados. Portanto, é necessário desenvolver pesquisas randomizadas com grupo controle e desenho metodológico de qualidade para avaliar a eficácia da auriculoterapia em pacientes com queixa de zumbido.

Palavras chave: Acupuntura. Ansiedade. Orelha externa. Qualidade de vida.

\footnotetext{
${ }^{1}$ Dentista, Doutora em Biotecnologia, mestre em Psicobiologia, especialista em Anatomia Patológica, Psicobiologia e Acupuntura. Professora de Neuroanatomia da Universidade Federal da Paraíba (UFPB). analucarneiro@gmail.com.

${ }^{2}$ Acadêmica do $6^{\circ}$ período do curso de Fonoaudiologia da UFPB. mariananadm@gmail.com.

3 Médico, especialista em Neurologia Clínica e Saúde da Família. Plantonista do Hospital de Emergência e Trauma Senador Humberto Lucena, Joao Pessoa. alveslindair@gmail.com.
} 
ABSTRACT: OBJECTIVE: To verify the action of the auriculotherapy in patients with tinnitus complaint. METHOD: Intervention study, with evaluations carried out pre and post-auriculotherapy sessions, presented as a case study in three patients with tinnitus complaint. Evaluations were conducted with the Tinnitus Handicap Inventory (THI), the Visual Analog Scale (VAS), the State - Trait Anxiety Inventory (STAI) and the Beck Anxiety Inventory (BAl) before and after the eight auriculotherapy sessions. The auricular evaluation was carried out by visual inspection, electrodiagnosis and palpation. After the energetic and auricular diagnosis, the combination of points was determined for each patient. In all the protocols were included the Shen men, kidney, inner ear, external ear, brainstem, thalamus and temporal points. To compose the individual protocol, other points were selected considering the tinnitus as a multifactorial symptom and the integral patient care. RESULTS: The data collected in the inventories and in the patients reports demonstrated that the auriculotherapy reduced the symptoms of tinnitus and anxiety and improved the quality of life of the patients. CONCLUSION: The auriculotherapy is an integral care technology which alters the perception of the tinnitus and improves the quality of life of the patients, proving to be a promising and efficient intervention in the evaluated cases. Therefore, it is necessary to develop randomized researches with control group and quality methodological design to evaluate the efficiency of the auriculotherapy in patients with tinnitus complaint.

Keywords: Acupuncture. Anxiety. The external ear. Quality of life. 


\section{INTRODUÇÃO}

O zumbido, também denominado acúfeno ou tinido, é um sintoma otoneurológico definido como a percepção consciente de sons ou ruídos na ausência de um estímulo sonoro externo. O som "fantasma" é referido como um som semelhante a apito, pulsação do coração, sinos, campainha, barulho de chuva ou ondas do mar e som de escape de panela de pressão, por exemplo. Essa ilusão sonora apresenta maior prevalência em idosos (VINAGRE; GUARIENTO, 2018; RUBEM CRUZ; ROGÉRIO POLI, 2007; SWAIN; NAYAK; RAVAN; SAHU, 2016).

Estudos demonstram que o zumbido possui uma correlação com outros transtornos neurológicos e psiquiátricos, tais como: cefaleia, insônia, ansiedade, depressão e labirintopatias. Dessa forma, o desconforto, a gravidade e suas consequências podem alterar as atividades de vida diária, causar o afastamento do convívio social, comprometer significativamente a qualidade de vida e, por fim, em casos extremos, levar o sujeito ao suicídio. Este desconforto é apontado como um dos três piores sintomas para o indivíduo, sendo superado apenas pelas dores e tonturas demasiadas e sem tratamento (VINAGRE; GUARIENTO, 2018; ROSA; ALMEIDA; PIMENTA; SILVA et al., 2012).

Esse transtorno auditivo "fantasma" é multifatorial, com fisiopatologia complexa e considerado um desafio para a equipe da saúde, pois até o momento não há um tratamento efetivo para eliminar o zumbido. A abordagem clínica do zumbido envolve associação de tratamentos farmacológico e não farmacológico que visam, em geral, minimizar o sintoma. Os métodos terapêuticos incluem Terapia Cognitivo Comportamental (TCC), psicoterapia, terapia da habituação ou Terapia de Retreinamento do Zumbido e Hipersensibilidade (Tinnitus Retraining Therapy - TRT), mascaramento, cirurgia, neurofeedback (GÜNTENSPERGER; THÜRING; MEYER; NEFF et al., 2017; HOLDEFER; OLIVEIRA; VENOSA, 2010; RUBEM CRUZ; ROGÉRIO POLI, 2007) e acupuntura (KIM; CHOI; LEE; CHOI et al., 2012; 
NADERINABI; SOLTANIPOUR; NEMATI; SABERI et al., 2018; XIE; LI; LAI; ZHOU et al., 2014).

As Práticas Integrativas e Complementares em Saúde (PICS) contém recursos terapêuticos indicados no tratamento do zumbido e a acupuntura é uma das opções mais comuns. O que diz a acupuntura sobre o zumbido? Na acupuntura o zumbido é um sintoma descrito, principalmente, nos desequilíbrios do meridiano do rim (deficiência no jing dos rins, deficiência do yin ou yang do rim) e dentre os fatores etiológicos encontram-se o excesso sexual, a idade avançada e as doenças crônicas (LIMA; RAPOSO, 2018; ROSS, 2011; AUTEROCHE e NAVAILH, 1992). Wang e Pai (2005) relacionaram o zumbido com o excesso de atividades sexuais, incluindo promiscuidade sexual, doenças sexualmente transmissíveis, infecção vaginal e multiparidade. Isto posto, transcreve-se a descrição dos autores Wang e Pai (2005) sobre o zumbido:

\begin{abstract}
A imoderação na vida sexual pode exaurir e debilitar o Jing do Shen, causando lassidão na região lombar e nos joelhos, vertigem, zumbido e hipoacusia. Além desses sintomas, os homens podem apresentar emissão seminal e ejaculação precoce, e as mulheres, anemia e leucorréia.
\end{abstract}

Ainda vale ressaltar o que diz Chenggu (2006) ao incluir o zumbido entre as deficiências do sangue no coração e no fígado, para ele a deficiência no sangue dificulta sua circulação para a região da cabeça e consequentemente surge palidez na face, enjoo e zumbido.

Muitas pesquisas foram realizadas para avaliar a eficácia da acupuntura sistêmica e da cranioacupuntura no tratamento do zumbido (DOI; TANO; SCHULTZ; BORGES et al., 2016; OKADA; ONISHI; CHAMI; BORIN et al., 2006; VERA; GRILLO; FORTINGUERRA; SOUSA et al., 2013) e verificaram melhora nos sintomas do zumbido e na qualidade de vida dos pacientes. Entretanto, pesquisas para verificar a efetividade da auriculoterapia em pacientes com zumbido são escassas, com pequenas amostras e associado a outros tratamentos (SHALADI; CRESTANI; SALTARI, 2009).

A acupuntura auricular ou auriculoterapia é uma tecnologia de cuidado que utiliza a orelha externa no diagnóstico energético e tratamento. Essa terapia de 
microssistema promove a conexão entre a orelha e o Sistema Nervoso Central (SNC) através dos estímulos realizados nos pontos auriculares com agulhas, esferas (prata, ouro, cristal, zircônio), sementes, stiper auricular ou laser. O estímulo auricular gera reflexos, produz reações que harmonizam e equilibram o organismo e promove, portanto, a regulação psíquico-orgânica do indivíduo (OLESON, 2013; DAL MAS, 2004; GARCÍA, 2003). Nesse sentido, o objetivo deste trabalho é relatar a ação da auriculoterapia em três pacientes com queixa de zumbido.

\section{MÉTODO}

Trata-se de um estudo de intervenção, com abordagem pré e pós experimental, apresentado como estudo de caso. A amostra foi constituída por três pacientes com queixa de zumbido, idade entre 54 e 72 anos, encaminhados pelo Grupo de Estudo e Pesquisa em Audição e Zumbido (GEPAEZ) da Universidade Federal da Paraíba (UFPB) para o atendimento de Auriculoterapia, realizado pelo projeto NeuroConexões: saúde, bem-estar e práticas integrativas e complementares. A equipe do projeto GEPAEZ investigou relatos de zumbido, acolheu os pacientes e realizou a avaliação inicial. Na primeira consulta a equipe fez acufenometria e audiometria. Além disso, foram aplicados os questionários: a) Tinnitus Handicap Inventory (THI), composto por 25 questões que abordam aspectos funcionais, emocionais e catastróficos do zumbido. Cada questão apresenta três possibilidades de resposta (sim, às vezes, não), cuja pontuação (0 a 100) pode categorizar o grau de gravidade do zumbido. O THI é um instrumento confiável para verificar o prejuízo causado pelo zumbido na qualidade de vida; b) Escala Visual Analógica (EVA), com pontuação de 0 a 10, que determina, de forma subjetiva, o volume e nível do incômodo do zumbido (AZEVEDO; OLIVEIRA; SIQUEIRA; FIGUEIREDO, 2007; FERREIRA; CUNHA; ONISHI; BRANCO-BARREIRO et al., 2005; FIGUEIREDO; DE AZEVEDO; OLIVEIRA, 2009). c) Inventário de Ansiedade Traço-Estado (IDATE), com escore entre 20 e 80 pontos, para quantificar elementos subjetivos relacionados a ansiedade. Nessa etapa foi aplicada o IDATE-T que avalia o traço de ansiedade, 
considerado mais estável quanto a propensão individual de lidar com maior ou menor ansiedade no decorrer da vida (FIORAVANTI et al. 2006). Os mesmos procedimentos foram utilizados para reavaliar os pacientes após oito sessões de auriculoterapia.

Este estudo foi aprovado pelo Comitê de Ética em Pesquisa (CEP), CCS/UFPB, parecer número 3.608.356 e todos os participantes assinaram o Termo de Consentimento Livre e Esclarecido (TCLE).

Antes da avaliação auricular com a equipe do projeto NeuroConexõesUFPB os voluntários preencheram o inventário de ansiedade Beck (Beck Anxiety Inventory$\mathrm{BAI}$ ) com 21 questões. O BAI é uma escala psicométrica de auto relato que verifica a intensidade de sintomas de ansiedade durante a última semana (CUNHA, 2017).

A avaliação auricular foi realizada por inspeção visual, eletrodiagnóstico, com aparelho localizador e estimulador EL 30, e palpação com apalpador de Nogier. Essa abordagem permite verificar alterações morfológicas na orelha (cor, nódulos, depressões, erupções) e também os pontos reagentes, aqueles sensíveis a pressão ou com alteração de resistência.

Após diagnóstico energético e auricular foi determinado a combinação de pontos para cada paciente. Em todos os protocolos foram incluídos os pontos Shen men, rim, ouvido interno, ouvido externo, tronco cerebral, tálamo e temporal (Figura 1). Os demais pontos foram selecionados considerando o zumbido um sintoma multifatorial, os pontos reagentes e o cuidado integral do paciente.

As sessões de intervenção ocorreram no ano de 2019, o protocolo auricular foi aplicado em uma orelha por vez. Foram utilizados algodão, álcool $70^{\circ}$, pinças, esferas com adesivo e agulha ting $(0,18 \times 8 \mathrm{~mm})$ com aplicador. As sessões foram realizadas uma vez por semana, durante dois meses, totalizando oito sessões. Os participantes foram orientados para estimular as respectivas regiões, pontos auriculares, três vezes ao dia. 


\section{Descrição dos Casos}

Paciente 1: Paciente com 54 anos, casado, $55 \mathrm{Kg}$, servidor público, apresentou queixa de zumbido com som de cigarra há aproximadamente um ano. $\mathrm{O}$ paciente relatou tontura, desconforto abdominal, frio nas mãos, pouca sede e baixo consumo de água. Durante a auriculomassoterapia queixou-se de dor na região do antitrago, principalmente na orelha direita. O diagnóstico auricular revelou vários pontos reagentes na região do antitrago e lóbulo da orelha (Figura 1).

A equipe GEPAEZ UFPB ao acolher o paciente e realizar o primeiro atendimento observou EVA de 2, escore THI de 22 pontos (grau leve de interferência na qualidade de vida) e IDATE com 39 pontos. O paciente apresentou nível mínimo de ansiedade $(\mathrm{BAl}=3)$ na primeira sessão de auriculoterapia.

Após oito sessões de auriculoterapia, na reavaliação, os resultados obtidos foram: EVA de 3, THI de 10 pontos (grau desprezível de interferência na qualidade de vida) e IDATE com 32 pontos (Tabela 1). Os resultados demonstraram um impacto positivo da auriculoterapia na qualidade de vida do paciente.

Paciente 2: Paciente com 72 anos, casado, $68 \mathrm{Kg}$, aposentado, músico, com zumbido bilateral há aproximadamente 24 anos. O paciente tinha indicação para uso de aparelho auricular, mas não utiliza devido a estética. Na avaliação energética o paciente relatou refluxo, faringite e gastrite. Para ele, o zumbido com som de cigarra é um "indicador emocional". Paciente faz uso de antidepressivo. No procedimento de auriculomassoterapia relatou dor na região do antitrago na orelha esquerda. $\mathrm{Na}$ segunda sessão relatou melhora em relação ao volume do zumbido. Na quinta sessão, referiu perceber diminuição de frequência e intensidade do zumbido, segundo ele "antes o som estava mais brabo". Na sétima sessão, decidiu utilizar o aparelho auditivo. $\mathrm{Na}$ oitava sessão, referiu que o zumbido estava suave, baixou o volume, pois "antes era mais agressivo".

Obteve como resultado na avaliação inicial os seguintes valores: EVA de 3, escore de THI de 12 pontos (grau desprezível de interferência na qualidade de vida), 
BAl com escore 3 (nível mínimo) e IDATE com 32 pontos. Enquanto na reavaliação, os resultados encontrados foram: EVA de 2, THI e IDATE se mantiveram (Tabela 1).

Paciente 3: Paciente com 63 anos, $50 \mathrm{Kg}$, aposentada, queixa-se de zumbido com som de cigarra e cachoeira, artrose em membros superiores (mãos e cotovelos), insônia e frequente emoção de raiva. Na avaliação auricular observou-se escamações, cravos, manchas acastanhadas na orelha e uma depressão no ponto ouvido externo (Figura 1). Durante procedimento de auriculomassoterapia a paciente apresentou desconforto na região do antitrago. A avaliação auricular identificou muitos pontos reagentes no lóbulo da orelha, no antitrago e na concha da orelha (Figura 1). Na terceira sessão a paciente relatou melhora no intestino. Na quinta sessão, observou redução no volume do zumbido e melhora no sono. $\mathrm{Na}$ reavaliação, após auriculoterapia, observou-se redução no EVA de 8 para 6, no escore THI de 66 (grau severo de interferência) para 48 (grau moderado de interferência na qualidade de vida), BAI de 17 (nível moderado de ansiedade) para 1 e depois para 7 (nível mínimo de ansiedade) e IDATE com 45 pontos, sem alterações.

A paciente declarou-se satisfeita com o tratamento e compartilhou da seguinte forma sua experiência: "Muito otimista com o tratamento do zumbido, já vejo resultados positivos no ouvido esquerdo. É impressionante como o desconhecido gera medo. Eu morria de medo de fazer acupuntura. Hoje ela é indicada pra cura do zumbido. Obrigada a esse grupo integrado e responsável da UFPB".

\section{RESULTADOS}

$\mathrm{Na}$ avaliação auricular os três pacientes relataram dor na região do antitrago e apresentaram similaridade de pontos com reação positiva, principalmente nas regiões do antitrago e lóbulo da orelha. Os pontos, frontal, temporal, occipital, tálamo e ouvido externo foram reativos nos três pacientes (Figura 1). Na paciente 3 , o ponto ouvido externo apresentou uma depressão, variação morfológica mais comum em pacientes com zumbido (AKAMINE et al., 2013). 
Os pontos auriculares reagentes nos três pacientes (Figura 1) são indicados para diversos transtornos otoneurológicos. O ponto occipital, por exemplo, é indicado para vertigem, tontura e doença de Ménière e o ponto ouvido externo, para zumbido e hipoacusia. A estimulação do ponto temporal regula a atividade do ramo coclear do nervo vestibulococlear (VIII par de nervo craniano) (GARCIA, 2003).

Quanto ao protocolo previamente estabelecido (Figura 1) seus pontos seguem o caminho neural da via auditiva e, portanto, inclui pontos relacionados as orelhas (ouvido interno e externo), o ponto tronco cerebral, tálamo e temporal. Outros pontos foram incluídos considerando os princípios da acupuntura. O ponto Shen Men ou sistema nervoso central, conhecido como porta da alma, é o mais frequente nas pesquisas com estresse e ansiedade. Além disso, os ponto Shen men e tronco cerebral tem propriedades calmantes enquanto o ponto rim tem função energética (DO PRADO; KUREBAYASHI; DA SILVA, 2018; KUREBAYASHI; GNATTA; BORGES; BELISSE et al., 2012; KUREBAYASHI; TURRINI; DE SOUZA; MARQUES et al., 2017).

A auriculoterapia demonstrou resultados positivos quando comparadas às avaliações nos momentos pré e pós intervenção. Os resultados do THI demonstraram melhora de dois pacientes quanto aos aspectos do zumbido (Tabela 1). Outras pesquisas demonstraram melhora significativa do zumbido em pacientes tratados com acupuntura e avaliados com o THI (DOI; TANO; SCHULTZ; BORGES et al., 2016; OKADA; ONISHI; CHAMI; BORIN et al., 2006).

Os dados coletados demonstraram que a auriculoterapia melhorou a qualidade de vida e os sintomas de ansiedade dos pacientes avaliados (Tabela 1). Para isso, utilizou-se como recurso a aplicação da escala EVA e dos inventários THI, IDATE-T e BAI. Esses protocolos apresentam boa confiabilidade, ratificada por altas consistências internas, facilidade, rapidez e reprodutibilidade de aplicação. Dessa forma, O uso das escalas, principalmente THI e EVA, nas pesquisas clínicas com zumbido tornam os resultados mais confiáveis. A EVA é um método simples, rápido e de fácil compreensão, entretanto, pode além de induzir uma avaliação superficial e variável sofrer influências culturais e do estado emocional do paciente e deve, portanto, ser associada a outros métodos (AZEVEDO; OLIVEIRA; SIQUEIRA; 
FIGUEIREDO, 2007; FIGUEIREDO; DE AZEVEDO; OLIVEIRA, 2009), conforme realizado no presente trabalho.

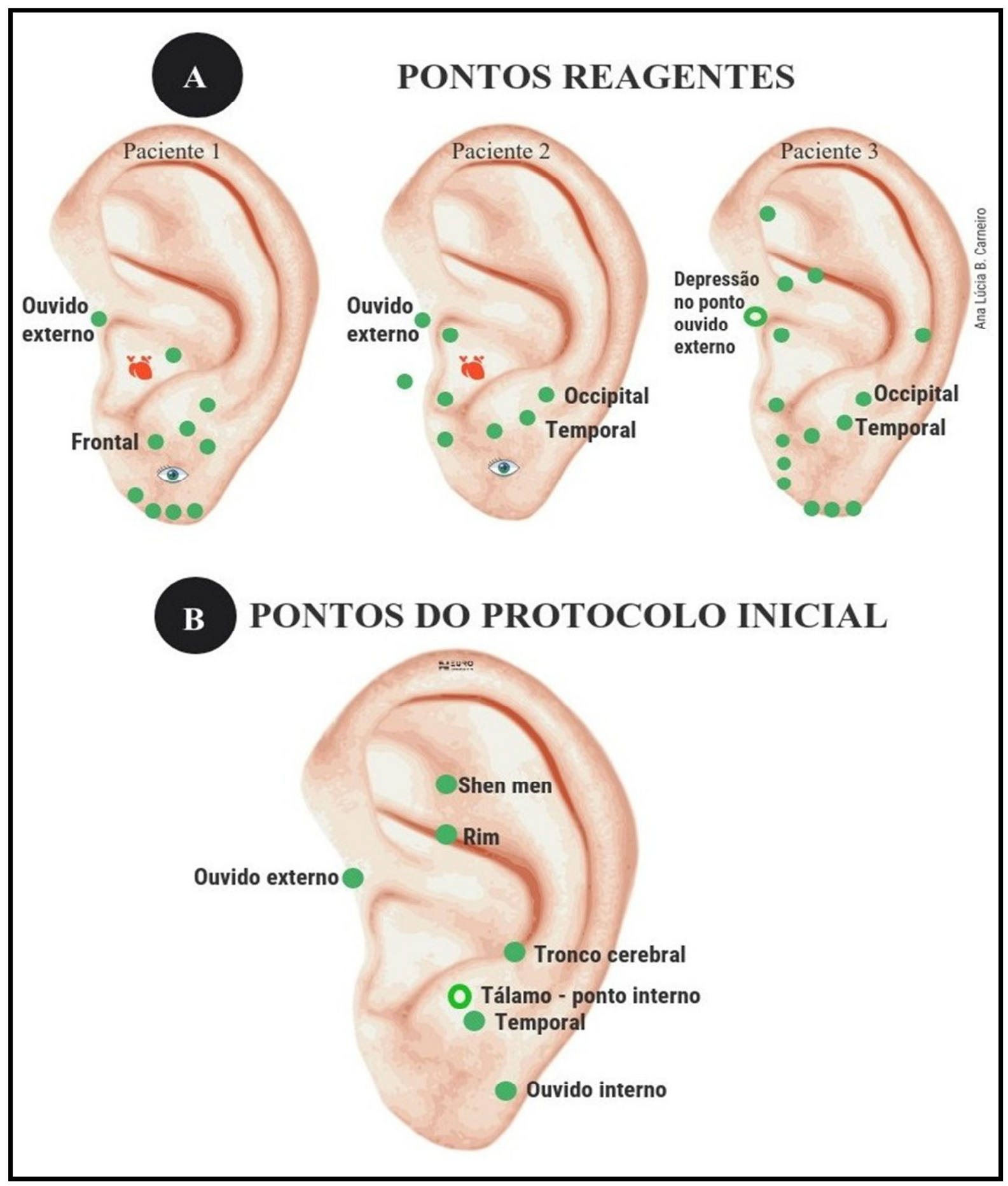

Figura 1. A: Pontos reagentes na avaliação auricular dos pacientes com queixa de zumbido. Observe a semelhança entre os pontos reagentes na região do lóbulo da orelha e antitrago.B: Pontos auriculares utilizados em todos os pacientes nas oito sessões de auriculoterapia. 
Tabela 1. Resultados obtidos nas avaliações no tempo 0 e nos momentos pré e pós intervenção com auriculoterapia.

\begin{tabular}{|c|c|c|c|}
\hline AVALIAÇÕES & PACIENTE 1 & PACIENTE 2 & PACIENTE 3 \\
\hline EVA 0 - Tempo 0 - GEPAEZ & 2 & 3 & 8 \\
\hline EVA $1-1^{a}$ sessão de auriculoterapia & $3-4$ & 6 & 8 \\
\hline $\begin{array}{llll}\text { EVA } 2 \text { - pós intervenção } & \text { com } \\
\text { auriculoterapia } & & & \\
\end{array}$ & 3 & 2 & 6 \\
\hline THI 0 - Tempo 0 - GEPAEZ & 22 & 12 & 66 \\
\hline $\begin{array}{lcccc}\text { THI } 1 & \text { - pós intervenção } & \text { com } \\
\text { auriculoterapia } & \text { - GEPAEZ } & \\
\end{array}$ & 10 & 12 & 48 \\
\hline IDATE 0 - Tempo 0 - GEPAEZ & 39 & 32 & 45 \\
\hline $\begin{array}{l}\text { IDATE 1- pós intervenção } \\
\text { auriculoterapia - GEPAEZ }\end{array}$ & 32 & 32 & 45 \\
\hline BAI 1- 1ª sessão de auriculoterapia & 3 & 3 & 17 \\
\hline BAI 2 - $5^{\text {a }}$ sessão de auriculoterapia & 2 & 1 & 1 \\
\hline BAI 3 - $8^{a}$ sessão de auriculoterapia & 1 & 0 & 7 \\
\hline
\end{tabular}

Para além dos inventários e seus escores estão a auto percepção do paciente sobre o seu estado e, nessa perspectiva, os relatos espontâneos demonstraram satisfação dos pacientes com a técnica e seus resultados.

Em decorrência dos resultados obtidos, pode-se afirmar que houve uma melhora dos pacientes, desde a diminuição na intensidade do zumbido até a melhoria na qualidade de vida como demonstrado por meio dos relatos e dados colhidos e apresentados na Tabela 1.

Acredita-se que a melhora progressiva dos sintomas esteja relacionada com padrões de equilíbrio global do paciente, características físicas e, principalmente, emocionais. Na prática clínica, a acupuntura e respectivas terapias de microssistemas (auriculoterapia, cranioacupuntura) são uma opção para a melhoria do bem-estar desses pacientes, pois sua eficácia clínica nos sintomas, principalmente emocionais, é maior do que de outros tipos de tratamento. Por fim, a acupuntura, principalmente a auriculoterapia, representa uma forma de cuidado integral de baixo custo e eficaz na melhora da qualidade de vida dos pacientes. 


\section{CONCLUSÃO}

Conclui-se, portanto, que a auriculoterapia é uma tecnologia de cuidado integral que altera a percepção do zumbido, diminui os sintomas de ansiedade e melhora a qualidade de vida dos pacientes, mostrando-se uma intervenção eficiente para o cuidado integral e promissora no acompanhamento de pacientes com zumbido. Portanto, é necessário desenvolver pesquisas randomizadas com grupo controle e desenho metodológico de qualidade para avaliar a eficácia da auriculoterapia no tratamento de pacientes com queixa de zumbido.

\section{REFERÊNCIAS BIBLIOGRÁFICAS}

AUTEROCHE, B.; NAVAILH, P. O Diagnóstico na Medicina Chinesa. São Paulo: Organização Andrei Editora, 1992.

AKAMINE, A. A. et al. Inspeção visual do pavilhão auricular em indivíduos com zumbido e/ou tontura: abordagem pela Medicina Tradicional Chinesa. Revista Equilíbrio Corporal e Saúde, v. 5, n. $2,2015$.

AZEVEDO, A. A. D.; OLIVEIRA, P. M. D.; SIQUEIRA, A. G. D.; FIGUEIREDO, R. R. Análise crítica dos métodos de mensuração do zumbido. Revista Brasileira de Otorrinolaringologia, 73, n. 3, p. 418-423, 2007.

CHENGGU, Ye. Tratamento das Doenças Mentais por Acupuntura e Moxabustão. São Paulo: Editora Roca, 2006.

CUNHA, J. A. Manual da Versão em Português das Escalas Beck. São Paulo: Pearson Clinical Brasil, 2017.

DAL MAS, W. D. Auriculoterapia-Auriculomedicina na Doutrina Brasileira. Editora Roca, 2004. 857241553X.

DO PRADO, J. M.; KUREBAYASHI, L. F. S.; DA SILVA, M. J. P. Auriculoterapia verdadeira e placebo para enfermeiros estressados: ensaio clínico randomizado. Rev Esc Enferm USP, 52, p. e03334, 2018.

DOI, M. Y.; TANO, S. S.; SCHULTZ, A. R.; BORGES, R. et al. Effectiveness of acupuncture therapy as treatment for tinnitus: a randomized controlled trial. Brazilian Journal of Otorhinolaryngology, 82, n. 4, p. 458-465, 2016.

FERREIRA, P. É. A.; CUNHA, F.; ONISHI, E. T.; BRANCO-BARREIRO, F. C. A. et al. Tinnitus Handicap Inventory: adaptação cultural para o português brasileiro. Pró-Fono Revista de Atualização Científica, 2005. 
FIGUEIREDO, R. R.; DE AZEVEDO, A. A.; OLIVEIRA, P. D. M. Análise da correlação entre a escala visual-análoga e o Tinnitus Handicap Inventory na avaliação de pacientes com zumbido. Brazilian Journal of Otorhinolaryngology, 75, n. 1, p. 76-79, 2009.

FIORAVANTI, A. C. M. et al. Avaliação da estrutura fatorial da escala de ansiedade-traço do IDATE. Avaliação Psicológica, v. 5, n. 2, p. 217-224, 2006.

GARCÍA, E. G. Auriculoterapia-Escola Huang Li Chun. Editora Roca, 2003. GÜNTENSPERGER, D.; THÜRING, C.; MEYER, M.; NEFF, P. et al Neurofeedback for Tinnitus Treatment - Review and Current Concepts. Frontiers in Aging Neuroscience, 9, p. 386, 2017.

HOLDEFER, L.; OLIVEIRA, C. A. C. P. D.; VENOSA, A. R. Sucesso no tratamento do zumbido com terapia em grupo. Brazilian Journal of Otorhinolaryngology, 76, n. 1, p. 102-106, 2010.

KIM, J.-I.; CHOI, J.-Y.; LEE, D.-H.; CHOI, T.-Y. et al. Acupuncture for the treatment of tinnitus: a systematic review of randomized clinical trials. BMC Complementary and Alternative Medicine, 12, n. 1, p. 97, 2012.

KUREBAYASHI, L. F. S.; GNATTA, J. R.; BORGES, T. P.; BELISSE, G. et al. Aplicabilidade da auriculoterapia com agulhas ou sementes para diminuição de estresse em profissionais de enfermagem. Revista da Escola de Enfermagem da USP, 46, n. 1, p. 89-95, 2012.

KUREBAYASHI, L. F. S.; TURRINI, R. N. T.; DE SOUZA, T. P. B.; MARQUES, C. F. et al.Auriculoterapia para redução de ansiedade e dor em profissionais de enfermagem: ensaio clínico randomizado. Revista Latino-Americana de Enfermagem, 25, p. e2843, 2017.

LIMA, P.R.; RAPOSO, C. Zang Fu Revelando a Cara da Síndrome. Porto Alegre: Editora Zen, 2018.

NADERINABI, B.; SOLTANIPOUR, S.; NEMATI, S.; SABERI, A. et al. Acupuncture for chronic nonpulsatile tinnitus: A randomized clinical trial. Caspian Journal of Internal Medicine, 9, n. 1, p. 38-45, 2018.

OKADA, D. M.; ONISHI, E. T.; CHAMI, F. I.; BORIN, A. et al. O uso da acupuntura para alívio imediato do zumbido. Revista Brasileira de Otorrinolaringologia, 72, n. 2, p. 182-186, 2006.

OLESON, T. Auriculotherapy manual: Chinese and western systems of ear acupuncture. Elsevier Health Sciences, 2013. 0702035726.

ROSA, M. R. D. D.; ALMEIDA, A. A. F. D.; PIMENTA, F.; SILVA, C. G. et al. Zumbido e ansiedade: uma revisão da literatura. Revista CEFAC, 14, n. 4, p. 742-754, 2012.

ROSS, J.. Combinações dos Pontos de Acupuntura: a chave para o êxito clínico. São Paulo: Editora Roca, 2003.

RUBEM CRUZ, S.; ROGÉRIO POLI, S. Zumbido. Revista da Faculdade de Ciências Médicas de Sorocaba, 5, n. 2, p. 22-24, 2007.

SHALADI, A. M.; CRESTANI, F.; SALTARI, R. Auricular acupuncture plus antioxidants in the treatment of subjective tinnitus: a case series. (Case Series) (Report). Medical Acupuncture, 21, n. 2, p. 131, 2009.

SWAIN, S. K.; NAYAK, S.; RAVAN, J. R.; SAHU, M. C. Tinnitus and its current treatment- Still an enigma in medicine. Journal of the Formosan Medical Association, 115, n. 3, p. 139-144, 2016. 
VERA, R. M. D. L. T.; GRILLO, C. M.; FORTINGUERRA, M. L. B.; SOUSA, M. D. L. R. et al. Acupuntura no manuseio da dor orofacial e do tinido: Relato de caso. Revista Dor, 14, n. 3, p. 226-230, 2013.

VINAGRE, L. M; GUARIENTO, M. E.. Tratamento clínico de zumbido primário em adultos e idosos: revisão sistemática. Revista da Sociedade Brasileira de Clínica Médica, v. 16, n. 4, p. 249-254, 2018.

WANG, L. G.; PAl, Hong J. (Ed.). Tratado Contemporâneo de Acupuntura e Moxibustão. I. Fundamentos da Medicina Tradicional Chinesa. II. Diagnóstico e Tratamento. Editora CEIMEC, 2005.

WEILER, A. L.; BORBA, C. A. S.; FERREIRA, E. C. P. Auriculoterapia: tratamento do transtorno de ansiedade em mulheres na menopausa e climatério. Revista Pensamento Biocêntrico, $\mathrm{n}$. 18, p. 119-137, 2012.

XIE, H.; LI, X.; LAI, J.; ZHOU, Y. et al. Effectiveness of De Qi during acupuncture for the treatment of tinnitus: study protocol for a randomized controlled trial. Trials, v.15, n. 1, p. 397 , 2014. 